\title{
Musculoskeletal imaging of the inflammatory and degenerative joints: current status and perspectives
}

\author{
Fausto Salaffi ${ }^{1}$ (D) Marina Carotti ${ }^{2} \cdot$ Antonio Barile $^{3}$
}

Received: 22 January 2019 / Accepted: 11 February 2019 / Published online: 27 February 2019

(c) Italian Society of Medical Radiology 2019

This special issue of "La Radiologia Medica" focuses on the broad spectrum of musculoskeletal imaging, ranging from essential concepts for inflammatory (rheumatoid arthritis, spondyloarthritis, gout, and other crystal-induced arthropathies), noninflammatory (osteoarthritis, algoneurodystrophy) to multisystem and general pathologic conditions (such as large-vessel vasculitis) [1]. Musculoskeletal radiology continues to evolve rapidly, benefiting from advances as continually improving magnets, sequences in magnetic resonance imaging (MRI) as well as the broader application of plain radiographs, ultrasonography (US), and computed tomography (CT), or dual-energy CT (DECT) to the musculoskeletal system [2]. The articles included in this focused issue have been compiled with a goal to provide a valuable resource for residents, fellows, and practicing radiologists and rheumatologists in the scope of a shared interpretation of musculoskeletal imaging examinations. Both specialties have a duty to use imaging cost-effectively, to provide the requisite clinical information on diagnosis, prognosis, and serial assessment with the least risk and inconvenience to the patient. This can be best achieved by close working relationships between the specialties.

The lead article, "An Overview of Commonly Used Radiographic Scoring Methods in Rheumatoid Arthritis and Psoriatic Arthritis," is spearheaded by Fausto Salaffi,

Fausto Salaffi

fausto.salaffi@gmail.com

Marina Carotti

marina.carotti@gmail.com

Antonio Barile

antonio.barile@cc.univaq.it

1 Rheumatological Clinic, Ospedale "Carlo Urbani”, Università Politecnica delle Marche, Jesi, Ancona, Italy

2 Department of Radiology, Ospedali Riuniti, Università Politecnica delle Marche, Ancona, Italy

3 Department of Applied Clinical Science and Biotechnology, University of L'Aquila, L'Aquila, Italy
MD, Ph.D., and colleagues at the Rheumatological Clinic, Università Politecnica delle Marche, Ancona (Italy); it provides a focused guide for the scoring of plain radiographs in patients with rheumatoid arthritis (RA) and psoriatic arthritis (PsA). Radiographic progression is one of the most important outcome measures in RA and PsA clinical trials, because it reflects historical disease activity, is associated with loss of function over time, and can be reliably assessed [3]. These differ in the joints and features assessed. This results in differences in the scoring range, but also in the method of performance [4]. The various scoring systems for the two diseases are detailed in this issue.

The potential applications of US in rheumatology have been further increased with the dawn of the "third generation" US machines, equipped with very high-frequency probes $(>10 \mathrm{MHz})$. These can reach a spatial resolution of less than a tenth of a millimeter and make it possible to study the finest details of the smaller joints and hand tendons which are involved early on in RA [5-8]. In their article, "Ultrasound imaging in rheumatoid arthritis," Emilio Filippucci, MD, and colleagues at the Rheumatological Clinic, Università Politecnica delle Marche, Ancona (Italy), provide an overview of the main studies focusing on the value of US in the assessment of the patients with RA, and discussing the elementary lesions detectable by US (synovitis, bone erosion, cartilage damage, tenosynovitis, and tendon damage), the scoring systems currently available, and the scanning protocols in definite clinical settings (undifferentiated arthritis, early and long-standing RA).

Several sonographic abnormalities may be also observed in patients with osteoarthritis (OA) [9]. These include changes within cartilage, joint space widening resulting from fluid collection with or without synovial proliferation, and osteophytes. Loss of the thin, sharp contour of the superficial margin of the cartilaginous layer is one of the early features of OA. The US is exquisitely sensitive in detecting structural changes within different tissues and can reveal fibrillation and cleft formation in osteoarthritis. In 
their article, "Clinical utility and potential of ultrasound in osteoarthritis," Tadashi Okano, MD, and colleagues at the Orthopedic Surgery, Osaka City University Graduate School of Medicine, Osaka (Japan), describe the specific findings and utility of US for OA assessment.

In the last decades, the use of imaging guidance (notably US, but also CT and MRI) has emerged as an essential tool to perform several different kinds of interventions around bone and soft tissues; in their article "Interventional therapeutic procedures to treat degenerative and inflammatory musculoskeletal conditions: state of the art", Vito Chianca, MD, and colleagues at the IRCCS Istituto Ortopedico Galeazzi, Milano (Italy), review the current indications and results of the main therapeutic procedures in interventional musculoskeletal radiology for degenerative and inflammatory conditions.

Along with the development of novel drugs and therapies that are intended to alter the course of degenerative osteoarthritis, imaging plays a key role for the early detection and accurate assessment of joint status [10-12]. Federico Bruno, $\mathrm{MD}$, and colleagues at the Department of Biotechnology and Applied Clinical Sciences, University of L'Aquila, L'Aquila (Italy), in the article "New advances in MRI diagnosis of degenerative osteoarthropathy of the peripheral joints," provide an overview of the recent applications of advanced MRI techniques for the evaluation of degenerative joint changes (notably affecting the articular cartilage) that could have a great potential in the early diagnosis and treatment monitoring.

While conventional radiography and US remain a mainstay in rheumatology, MRI and CT have become increasingly common in patient care. Efficient methods for diagnosis, monitoring, and prognostication are essential in early RA [13, 14].

Mikkel Østergaard, MD, Ph.D., and colleagues at the Center for Rheumatology and Spine Diseases, Rigshospitalet, Glostrup, and the Department of Clinical Medicine, University of Copenhagen, Copenhagen (Denmark), focused on the potential uses of MRI and CT in the clinical management of patients with suspected or definite RA in the article "Imaging in Rheumatoid Arthritis: The Role of Magnetic Resonance Imaging and Computed Tomography." After sections on technical aspects, it described the current knowledge on MRI and CT for early detection of RA manifestations, diagnosis of RA, monitoring of disease activity and joint damage progression, and its role in determining prognosis.

Sacroiliac (SI) joints involvement is a crucial feature of inflammatory spondyloarthropathies, and MRI is the gold standard imaging modality to detect SI pathology [15-18]. Maria Antonietta Mazzei, MD, and colleagues at the Department of Medical, Surgical and Neuro Sciences, Diagnostic Imaging, University of Siena (Italy), in their article "Magnetic resonance imaging of the sacroiliac joints in SpA: with or without intravenous contrast media?", discuss the different MR imaging modalities and strategies available for the assessment of inflammatory involvement of the SI joints, with a particular focus on the advantages and limits in the use of intravenous contrast media.

Despite the recognized fundamental role of MRI in inflammatory joint pathologies, as previously discussed, MR imaging findings in clinical diagnostic criteria often lack specificity, giving potential diagnostic issues especially in the differential diagnosis with degenerative alterations [18-20]. To further explore this topic, in the contribution "MRI of the axial skeleton: differentiating non-inflammatory disease and axial spondyloarthritis - a review of current concept and applications," Ernesto La Paglia, MD, and colleagues at the Department of Radiology, Ospedale Ss. Antonio e Biagio e Cesare Arrigo, Alessandria (Italy), discuss the main MRI signs and the possible differential diagnoses, to improve the diagnostic performance in inflammatory spondyloarthropathies.

Although the role of imaging in the diagnosis, staging, and disease monitoring has been well established [21], several anatomical variants and imaging artifacts can show unusual appearance, leading to possible diagnostic pitfalls $[22,23]$. Marcello Zappia, MD, and colleagues at the Dipartimento di Medicina e di Scienze della Salute "Vincenzo Tiberio," Università del Molise, Campobasso (Italy), with their contribution "Diagnostic imaging pitfalls in rheumatology," illustrate the possible imaging pitfalls encountered in the imaging of inflammatory joint pathologies, and the available tips and tricks to recognize them.

With the assistance of innovations in scanner engineering and software design, dual-energy CT (DECT) is a recently developed advanced imaging method in the last decade. The principal advantages of DECT over conventional CT in the musculoskeletal setting relate to the additional information provided regarding tissue composition, artifact reduction, and image optimization [24-26]. Marina Carotti, MD, and colleagues at the Radiology Department, Università Politecnica delle Marche, Ancona (Italy), focus on applications of DECT to musculoskeletal imaging including gout and other crystal-induced arthropathies, virtual non-calcium images, and the study of bone marrow lesions in "The application of dual-energy computed tomography in the diagnosis of musculoskeletal disorders-A review of current concepts and applications."

The next portion of this monograph is dedicated to multimodality musculoskeletal imaging. This section of the monograph issue comprises a topic relevant to the practicing radiologist and rheumatologist. Two articles are devoted to the large-vessel vasculitis (LVV) and the utility of a US B-lines assessment as an adjunct method 
to assess interstitial lung disease (ILD) in patients with connective tissue disorders (CTD).

IPF is a frequent manifestation in patients with CTD [27-30]. Recently, the US criterion validity for its assessment has been proposed [27, 28]. In their article, "Ultrasound as a potential tool for the assessment of interstitial lung disease in rheumatic patients. Where are we now?", Marwin Gutierrez, MD, and colleagues at the Instituto Nacional de Rehabilitación, Mexico City, and Rheumatology Section, Center of Excellence of Rheumatology, Mexico City (Mexico), provide an overview of the role of US in the assessment of ILD in rheumatic disorders and discuss the current evidence supporting its clinical application in daily clinical practice.

LVV is the most common form of primary vasculitis comprising giant cell arteritis (GCA), Takayasu arteritis (TAK), and idiopathic aortitis (IA) [31, 32]. Early diagnosis and treatment of LVV are paramount to reduce the risk of ischemic complications such as visual loss and strokes, vascular stenosis and occlusion, and aortic aneurysm formation. Use of imaging modalities (US, MRI, CT, and [18F]-fluorodeoxyglucose positron emission tomography (PET)) has steadily increased to enable assessment of cranial and extracranial arteries, as well as the aorta. Key concepts underlying LVV are covered in the article "EULAR Recommendations for the use of Imaging in Large Vessel Vasculitis in Clinical Practice" by Mohammad Bardi, MD, and colleagues at the Department of Rheumatology, University of British Columbia (Canada), that summarizes the current evidence of imaging in patients with or suspected of having LVV and highlights the clinical implications of the EULAR recommendations.

As guest editors, we hope that this monograph issue provides residents, fellows, and practicing radiologists and rheumatologists with a strong foundation for interpreting multimodality musculoskeletal imaging studies. The numerous authors composing these articles have contributed their expertise with the intention of providing the most accurate and current information available at the time of publication; we are grateful for their incredible efforts.

\section{Compliance with ethical standards}

Conflict of interest The authors declare that they have no conflict of interest.

Ethical standards This article does not contain any studies with human participants or animals performed by any of the authors.

\section{References}

1. Colebatch AN, Edwards CJ, Østergaard M et al (2013) EULAR recommendations for the use of imaging of the joints in the clinical management of rheumatoid arthritis. Ann Rheum Dis 72(6):804-814

2. Østergaard M, Pedersen SJ, Døhn UM (2008) Imaging in rheumatoid arthritis-status and recent advances for magnetic resonance imaging, ultrasonography, computed tomography and conventional radiography. Best Pract Res Clin Rheumatol 22(6):1019-1044

3. Salaffi F, Carotti M, Ciapetti A, Gasparini S, Filippucci E, Grassi W (2011) Relationship between time-integrated disease activity estimated by DAS28-CRP and radiographic progression of anatomical damage in patients with early rheumatoid arthritis. BMC Musculoskelet Disord 30(12):120

4. Salaffi F, Carotti M, Di Donato E et al (2018) Preliminary validation of the simplified psoriatic arthritis radiographic score (SPARS). Skeletal Radiol. https://doi.org/10.1007/s0025 6-018-3124-0

5. Vreju FA, Filippucci E, Gutierrez M et al (2016) ultrasound synovitis in a particular joint is associated with ultrasound evidence of bone erosions in that same joint in rheumatoid patients in clinical remission. Clin Exp Rheumatol 34(4):673-678

6. Tămaş MM, Filippucci E, Becciolini A et al (2014) Bone erosions in rheumatoid arthritis: ultrasound findings in the early stage of the disease. Rheumatology (Oxford) 53(6):1100-1107

7. Carotti M, Salaffi F, Morbiducci J et al (2012) Colour Doppler ultrasonography evaluation of vascularization in the wrist and finger joints in rheumatoid arthritis patients and healthy subjects. Eur J Radiol 81(8):1834-1838

8. Filippucci E, Gabba A, Di Geso L, Girolimetti R, Salaffi F, Grassi W (2012) Hand tendon involvement in rheumatoid arthritis: an ultrasound study. Semin Arthritis Rheum 41(6):752-760

9. Okano T, Filippucci E, Di Carlo M et al (2016) Ultrasonographic evaluation of joint damage in knee osteoarthritis: feature-specific comparisons with conventional radiography. Rheumatology (Oxford) 55(11):2040-2049

10. Barile A, La Marra A, Arrigoni F et al (2016) Anaesthetics, steroids and platelet-rich plasma (PRP) in ultrasound-guided musculoskeletal procedures. Br J Radiol 89(1065):20150355

11. Li X, Majumdar S (2013) Quantitative magnetic resonance imaging of articular cartilage and its clinical applications. J Magn Reson Imaging 38:991-1008

12. Demehri S, Guermazi A, Kwoh CK (2016) Diagnosis and longitudinal assessment of osteoarthritis: review of available imaging techniques. Rheum Dis Clin North Am 42(4):607-620

13. Carotti M, Salaffi F, Di Carlo M, Sessa F, Giovagnoni A (2018) Magnetic resonance imaging of the craniovertebral junction in early rheumatoid arthritis. Skelet Radiol. https://doi.org/10.1007/ s00256-018-3055-9

14. Østergaard M, Peterfy C, Conaghan P et al (2003) OMERACT rheumatoid arthritis magnetic resonance imaging studies. Core set of MRI acquisitions, joint pathology definitions, and the OMERACT RA-MRI scoring system. J Rheumatol 30(6):1385-1386

15. Pedersen SJ, Weber U, Ostergaard M (2012) The diagnostic utility of MRI in spondyloarthritis. Best Pract Res Clin Rheumatol 26(6):751-766

16. Marzo-Ortega H, McGonagle D, Bennett AN (2010) Magnetic resonance imaging in spondyloarthritis. Curr Opin Rheumatol 22(4):381-387

17. Guglielmi G, Cascavilla A, Scalzo G, Carotti M, Salaffi F, Grassi W (2011) Imaging findings of sacroiliac joints in spondyloarthropathies and other rheumatic conditions. Radiol Med 116(2):292-301 
18. Guglielmi G, Scalzo G, Cascavilla A, Carotti M, Salaffi F, Grassi W (2009) Imaging of the sacroiliac joint involvement in seronegative spondylarthropathies. Clin Rheumatol 28(9):1007-1019

19. Lacout A, Rousselin B, Pelage JP (2008) CT and MRI of spine and sacroiliac involvement in spondyloarthropathy. AJR Am J Roentgenol 191(4):1016-1023

20. Rudwaleit M, Jurik AG, Hermann KG et al (2009) Defining active sacroiliitis on magnetic resonance imaging (MRI) for classification of axial spondyloarthritis: a consensual approac by the ASAS/ OMERACT MRI group. Ann Rheum Dis 68(10):1520-1527

21. Barile A, Arrigoni F, Bruno F et al (2017) Computed tomography and MR imaging in rheumatoid arthritis. Radiol Clin North Am 55(5):997-1007

22. McQueen F, Østergaard M, Peterfy C et al (2005) Pitfalls in scoring MR images of rheumatoid arthritis wrist and metacarpophalangeal joints. Ann Rheum Dis 64(SUPPL 1):48-55

23. Khanna S, Crues JV (2009) Complexities of MRI and false positive findings. Ann N Y Acad Sci 1154:239-258

24. Nicolaou S, Yong-Hing CJ, Galea-Soler S et al (2010) Dualenergy CT as a potential new diagnostic tool in the management of gout in the acute setting. Am J Roentgenol 194:1072-1078

25. Glazebrook KN, Guimarães LS, Murthy NS et al (2011) Identification of intraarticular and periarticular uric acid crystals with dual-energy CT: initial evaluation. Radiology 261:516-524

26. Choi HK, Al-Arfaj AM, Eftekhari A et al (2009) Dual energy computed tomography in tophaceous gout. Ann Rheum Dis 68:1609-1612

27. Tardella M, Di Carlo M, Carotti M, Filippucci E, Grassi W, Salaffi F (2018) Ultrasound B-lines in the evaluation of interstitial lung disease in patients with systemic sclerosis: cut-off point definition for the presence of significant pulmonary fibrosis. Medicine (Baltimore) 97(18):e0566

28. Gutierrez M, Gomez-Quiroz LE, Clavijo-Cornejo D et al (2016) Ultrasound in the interstitial pulmonary fibrosis. Can it facilitate a best routine assessment in rheumatic disorders? Clin Rheumatol 35(10):2387-2395

29. Salaffi F, Di Carlo M, Carotti M, Fraticelli P, Gabrielli A, Giovagnoni A (2018) Relationship between interstitial lung disease and oesophageal dilatation on chest high-resolution computed tomography in patients with systemic sclerosis: a cross-sectional study. Radiol Med 123(9):655-663

30. Salaffi F, Carotti M, Bosello S et al (2015) Computer-aided quantification of interstitial lung disease from high resolution computed tomography images in systemic sclerosis: correlation with visual reader-based score and physiologic tests. Biomed Res Int 2015:834262

31. Yamada I, Nakagawa T, Himeno Y et al (1998) Takayasu arteritis: evaluation of the thoracic aorta with CT angiography. Radiology 209:103-109

32. Diamantopoulos AP, Haugeberg G, Hetland H et al (2014) Diagnostic value of color Doppler ultrasonography of temporal arteries and large vessels in giant cell arteritis: a consecutive case series. Arthritis Care Res 66:113-119

Publisher's Note Springer Nature remains neutral with regard to jurisdictional claims in published maps and institutional affiliations. 